\title{
Functionally Generated Pathway To Harmonise Occlusion in Full Mouth Rehabilitation
}

\author{
Vyshnavi $\mathrm{Ch}^{1}$, Taruna $\mathrm{M}^{2}$, Sudhir $\mathrm{N}^{3}$, Chittaranjan $\mathrm{B}^{4}$
}

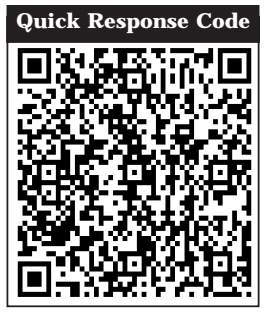

doi: $10.5866 / 2016.8 .10106$

1Post Graduate Student

2\&3Professor

${ }^{4}$ Professor \& HOD

Department of Prosthodontics

Kamineni Institute of Dental Sciences,

Narketpally, Nalgonda (Dt).

\section{Article Info:}

Received: April 12, 2016

Review Completed: May 13, 2016

Accepted: J une 11, 2016

Available Online: September, 2016 (www.nacd.in)

C NAD, 2016 - All rights reserved

\section{Email for correspondence:}

chunchuvyshnavi@gmail.com

\begin{abstract}
:
Planning and executing the restorative rehabilitation of a decimated occlusion is probably one of the most intellectually and technically demanding tasks facing a restorative dentist. Occlusal rehabilitation involves restoring the dentate or a partially dentate mouth. The aim of this report is to provide an orderly pattern of occlusal contact and articulation that will optimize oral function, occlusal stability and esthetics. The present article discuss a case report of full mouth rehabilitation using Panky Mann Schyuler technique with functionally generated path method.
\end{abstract}

Key words: Full mouth rehabilition, Ocdusion, Panky Mann Schyuler ,Functionally generated pathway.

\section{INTRODUCTION}

Prosthodontics deals with treatment and correction of defects by providing a pleasing appearance to the patient in addition to speech and mastication. Among the various prosthodontics procedures, full mouth rehabilitation is one of the most widely and common dental treatment for problems associated with dentinal hypersensitivity, esthetic corrections, loss in vertical dimension and problems in temperomandibular joint.

One of the most practical philosophies for occlusal rehabilitation is Pankey-Mann-Schuyler
Concept. The functionally generated path (FGP) is a static representation of the opposing cusp's dynamic movement from a centric position to eccentric positions to achieve optimal articulation and occlusal harmony. The FGP technique is normally used in the fabrication of maxillary posterior restorations with bilateral group function occlusion while liminating the nonworking sideand protrusive interferences.

Full mouth rehabilitation seeks to convert all unfavorable forces on teeth which invariably lead to periodontal conditions, into favorable forces which permit normal function and thereforeinduce healthy 
condition of thestomatognthic system. Dentist doing occlusal rehabilitation must be thorough and efficient with the principles of reconstruction. In full mouth rehabilitation there are different philosophies and concepts among them based on clinical situation of the patient prosthodontist chooses the apt technique. The present article discusses a full mouth rehabilitation case using Panky Mann Schuyler technique and functionally generated pathway

\section{CASE REPORT}

A patient of age $55 \mathrm{yrs}$ reported to the Department of the Prosthodontics, Kamineni Institute of Dental Sciences, Narketpally with a chief complaint of missing teeth and desired for the replacement to enhance his looks. Clinical and radiographic examination was carried out with a diagnosis of Chronic generalized periodontitis with bone loss and supraeruptions. Patient was referred to the periodontics department for improvement of the periodontal status. Full mouth periodontal flap surgery was advised and carried out which was followed up for 6 months and noticed marked improvement in the condition of the patient. As periodontal status stabilized full mouth rehabilitation was planned.

Patient does not have any medical history or previous dental history. On extraoral examination TMJ of the patient was clinically asymptomatic. Intraoral examination revealed that maxillary anterior teeth had gingival recession, and were malaligned with spacing between the teeth. Posterior teeth were severely attrited. It was noted that there was no loss of vertical dimension. 14 and 36 were missing and 38,48 and 41 were mobile.

\section{TREATMENT PLANNING}

After thorough clinical and radiological examination, it was planned for full mouth rehabilitation. Before proceeding with prosthetic phase patient was referred to endodontic department for full mouth intentional root canal treatment as most of his teeth were attrited and there was severe gingival recession. Further maxillary anteriors were extruded and required correction in the occlusal plane (Figure 1).

\section{PROCEDURE INVOLVED FOLLOWING STEPS:}

1. Diagnostic impressions were made using irreversible hydrocolloid impression material.

2. Facebow transfer was done using Hanau Spring Bow and was transferred to the Hanau Wide Vue articulator. Maxillary cast mounting was done accordingly. Mandibular mounting is done using interocclusal record.

3. A diagnostic mock wax up was done to design the cuspal angulations, occlusal planes and realign the teeth (Figure 2).

4. Anterior teeth and lower posterior teeth were prepared (Figure 3) and temporization was done by making an index of the mock wax up.

5. Final impressions were made using polyvinylsiloxane impression material. Casts were poured using die stone. Casts were mounted on to the Hanau wide vue using facebow transfer.

6. Wax patterns were fabricated as per the definitive values of temporaries, casting was done and the metal coping were tried in the patients mouth.

7. Bisque trial of upper and lower anteriors was done and permanent cementation was done using GIC luting cement after glazing of the prosthesis.

8. Later lower posterior teeth permanent cementation was done with GIC luting cement.

9. Upper posterior teeth were prepared and functionally generated pathway (FGP) impression was made using green stick compound (Figure 4) and temporization of the upper posterior teeth was done.

10. FGP impression was poured using die stone to record dynamic movements of theopposing cusps.

11. Mounting of the upper cast was done using facebow transfer and functional core was also mounted (Figure 5)

12. Wax pattern was fabricated and metal copings were tried in the patient mouth. 


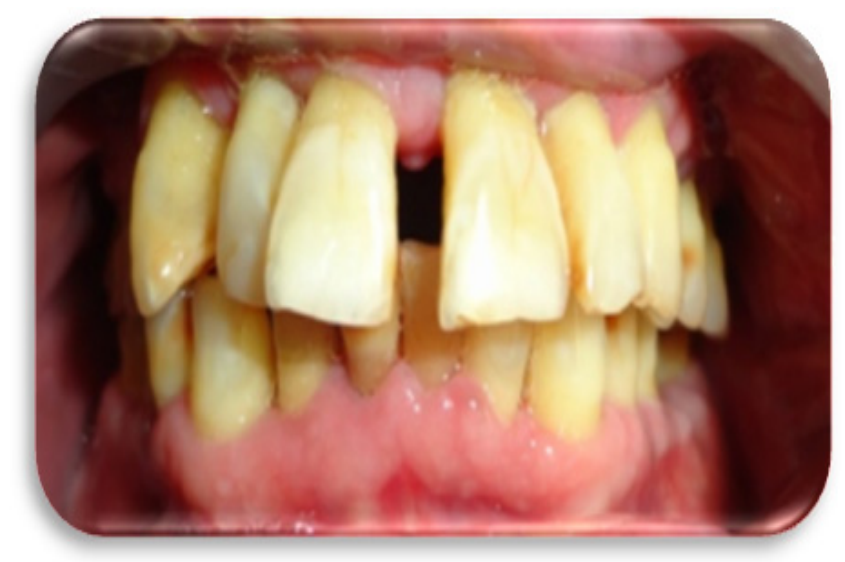

Figure 1: Preoperative view showing extruded maxillary anteriors

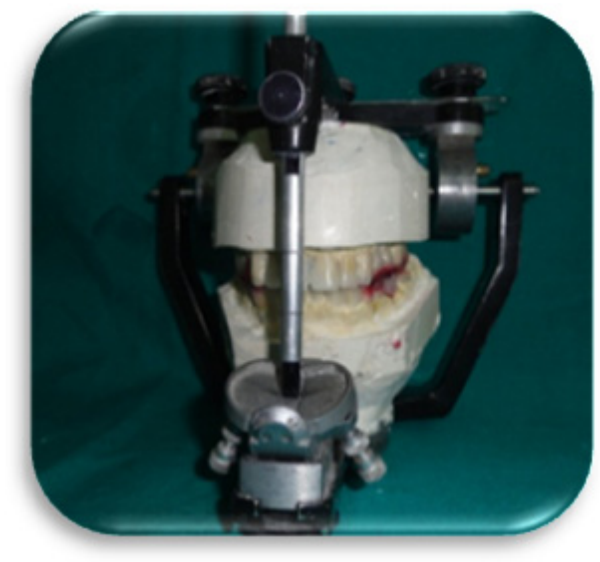

Figure 2: Diagnostic wax up
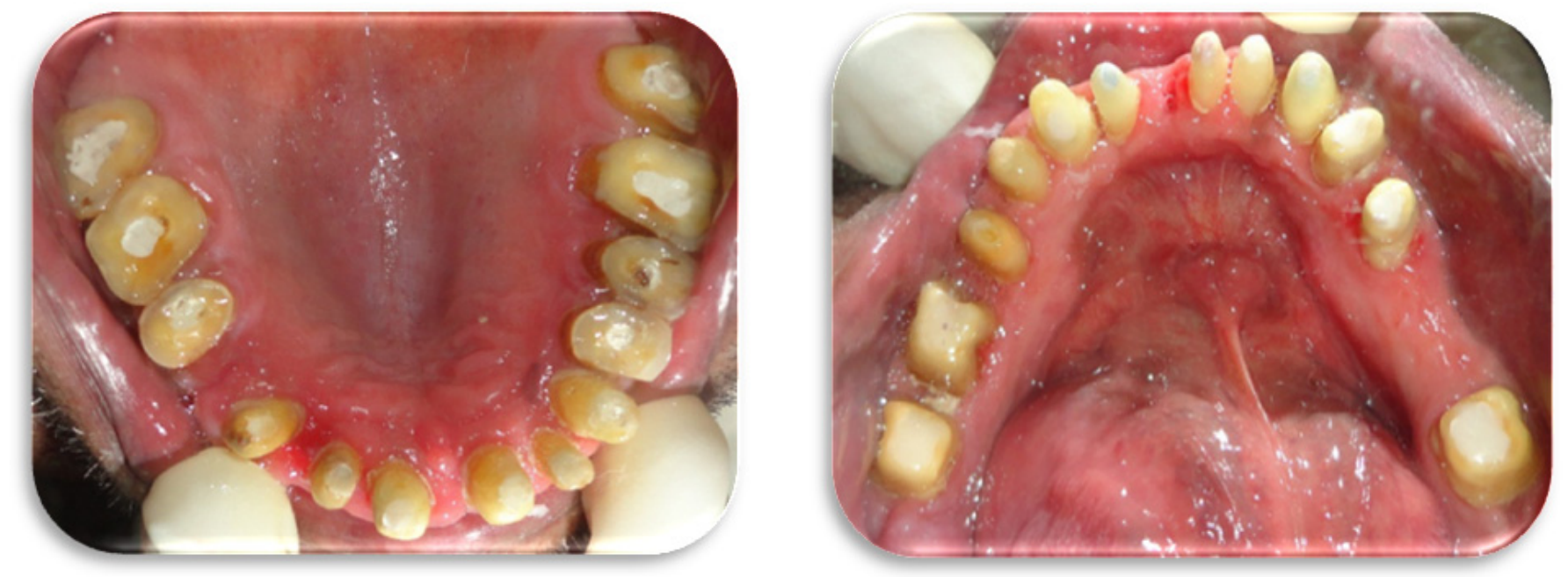

Figure3: Maxillary and mandibular teeth preparation

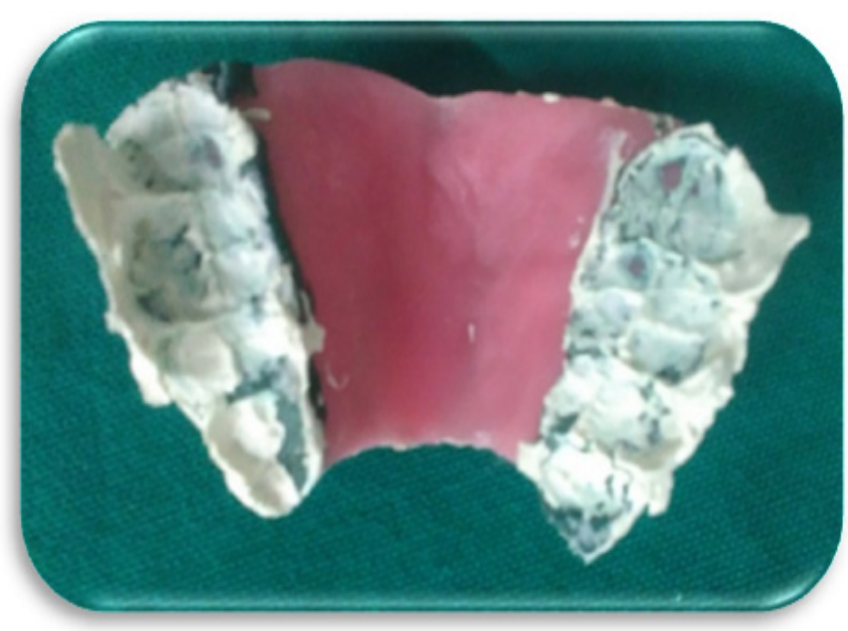

Figure 4: FGP impression

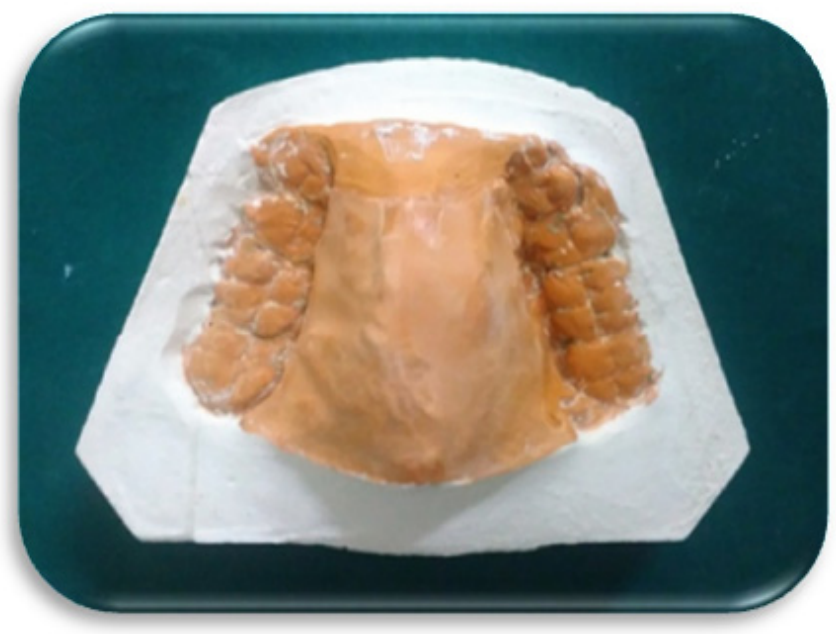

Figure 5: Functional core 

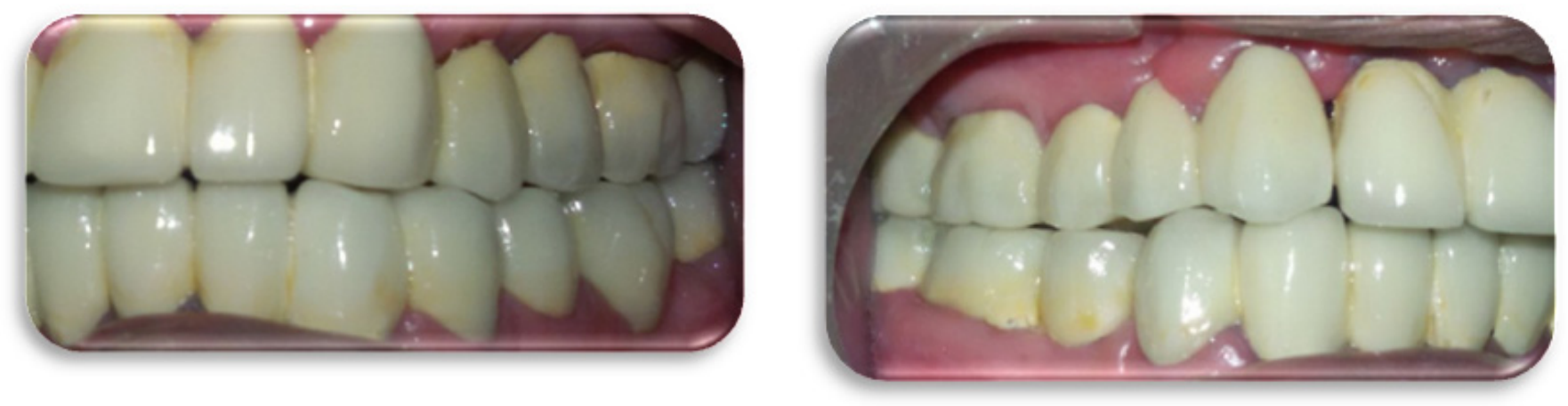

Figure 6: Final prosthesis with group function occlusion

13. Ceramic buildup was done and selective grinding was done on the articulator using FGP thus, reducing chair-side time.

14. Permanent cementation of upper posterior was done using GIC luting cement (Figure 6).

15. Oral hygiene maintenance instructions were given to the patient.

16. Follow up was carried out at regular intervals.

\section{DISCUSSION}

Full mouth rehabilitation is a challenging treatment modality that enhances the appearance of the patient and corrects imperfections in the occlusion. The most common reason for doing full mouth rehabilitation is to obtain and maintain the health of periodontal tissues. Abutment teeth are subjected to higher than usual occlusal forces transmitted through the prosthesis, the clinician must evaluate the abutment teeth carefully. Poor crown-to-root ratio can result from improper dental treatment as well as from traumatic or pathologic changes that increase the length of the clinical crown. However, the most common cause of poor (increased) crown-to-root ratio is periodontitis. The mobility seen in teeth with poor crown-to-root ratio can be reduced by selectively grinding occlusal surfaces and minimizing horizontal forces in the existing dentition. ${ }^{1}$ I $n$ the present case as there was poor crown root ratio with uneven occlusion plane and traumatic occlusion. It was planned for a confirmative approach of full mouth rehabilitation which met the mechanical and biological factors for long term success. The treatment not only focused on the esthetics and functional aspect of the dentition but also improved the health of the whole stomatognathic system of the patient. However for any case a detailed diagnosis and treatment planning is necessary to achieve predictable success.

After reviewing the various occlusal concepts, we are of the opinion that it is best to achieve posterior disocclusion in full mouth rehabilitation to avoid harmful lateral forces. ${ }^{2}$ One of the most practical philosophies is the Panky Mann Schuyler technique (PMS). However the selection of the full mouth rehabilitation technique solely depends on the clinical situation. For the technique used in this case report, has salient features as static coordinated occlusal contact of the maximum number of teeth to be present when the mandible is in centric relation. Further an anterior guidance has to be in harmony with function in lateral eccentric position on the working side. Also there has to be disocdlusion of all posterior teeth in protrusion by the influence of the anterior guidance. Disocclusion of all non-working inclines in lateral excursions and group function of the working side inclines in lateral excursions are the basic criteria to be met for the PMS concept. ${ }^{3}$

The technique for recording the FGP can be initiated after the anterior guidance has been harmonized according to the patients functional, esthetic, and periodontal support Functionally generated path procedures, used on upper posterior teeth, record directly all possible border pathways of the lower posterior teeth, as they are influenced by both theanterior and posterior determinants. The shape of the occlusal surfaces of the lower posterior teeth has a profound influence on the type of occlusion. The novel FGP technique described 
produces restorations with relatively normal occlusal anatomy, required minimal chairside occlusal adjustment, and were harmonious with the patient's existing bilateral group function occlusal scheme. ${ }^{4}$ When understood and appreciated, use of FGP technique is straight forward and practical method to achieve harmonious occlusal anatomy of restorations with the anterior determinant (anterior guidance), posterior determinant (condylar guidance), existing occlusal and cuspal anatomy, and the neuro-muscular system.

Dawson describes the FGP technique as an extremely sophisticated and practical method of capturing the precise border pathways that the lower posterior teeth follow. The technique has a distinct advantage in that it is able to record all dimensions of such border movements at the correct vertical as they are directly influenced by both condylar guidance and incisal guidance. ${ }^{5-7}$

\section{CONCLUSION}

The present technique of full mouth rehabilitation relies on patient anterior guidance, condylar guidance and functionally generated pathway. In the present case report, the patient suffered from severe disharmony in the crown root ratio, occlusal planeand occlusion so, group function type of occlusion was used in distributing the stresses to the posterior teeth, crown root ratio is altered without increasing the vertical dimension.
The choice of philosophy followed is depended on the case selected, operator knowledge and skills.

\section{REFERENCES}

1. Crown-to-root ratio: Its significance in restorative dentistry, J Prosthet Dent J uly 1979 Volume 42 Number 1.

2. Bhawana Tiwari, Komal Ladha, Dwarakananda Naik B, Aaruti Lalit. Occlusal Concepts in Full Mouth Rehabilitation: An Overview. J Indian Prosthodont Soc 2014.

3. Manoj Shetty, Krishna Prasad D, Niranjan J oshi, Sonali Sood. Complete Rehabilitation of a Patient with Occlusal Wear: A Case Report. J Indian Prosthodont Soc 2012; 12(3):191-197.

4. Nicholas B DuVall. Application of the Functionally Generated Path Technique to Restore Mandibular Molars in Bilateral Group Function Occlusion. J Prosthodontics 2013; 22: 226-232.

5. Gautam Bagchi, Archana Malviya, Gaurav Chandra, Meghna Goswami. Fabrication of posterior PFM restoration by functionally generated path technique using a modified recording base: A case report. IJ RID 2015; 5(6).

6. Rosenstiel RF, Land MF, Fujimoto J . Contemporary fixed prosthodontics. $1^{\text {st }}$ Ed., Mosby Publications, 1988.

7. Shillingburg HT, Hobo S, Whisett LD, J acobi R, Brackett SE. Fundamentals of fixed prosthodontics, $3^{\text {rd }} \mathrm{Ed}$., Quintessence Publication, 1997.

\section{Gain quick access to our journal online View our journal at www.nacd.in}

